\title{
Une approche féministe de l'accumulation primitive. Notes sur Caliban et la sorcière de Silvia Federici
}

\section{Öznur Karakass}

\section{OpenEdition}

\section{Journals}

Édition électronique

URL : http://journals.openedition.org/grm/783

DOI : $10.4000 / \mathrm{grm} .783$

ISSN : 1775-3902

\section{Éditeur}

Groupe de Recherches Matérialistes

Référence électronique

Öznur Karakass, « Une approche féministe de l'accumulation primitive. Notes sur Caliban et la sorcière de Silvia Federici », Cahiers du GRM [En ligne], 9 | 2016, mis en ligne le 30 juin 2016, consulté le 01 mai 2019. URL : http://journals.openedition.org/grm/783; DOI : 10.4000/grm.783

Ce document a été généré automatiquement le 1 mai 2019.

(c) GRM - Association 


\title{
Une approche féministe de l'accumulation primitive. Notes sur Caliban et la sorcière de Silvia Federici
}

\author{
Öznur Karakass
}

\section{Des modes de production}

1 Dans cet article j'aborderai brièvement l'approche féministe de l'accumulation primitive développée dans le livre de Silvia Federici intitulé Caliban and the Witch: Women, Body and Primitive Accumulation ${ }^{1}$. Cet ouvrage est le prolongement des études de Mariarosa Dalla Costa dans Women and the Subvertion of the Community (1971) et de Selma James dans Sex, Race and Class (1975) menées dans les années 1970 au sein du mouvement féministe, dans le but de rendre le travail domestique visible ${ }^{2}$.

2 Contre l'orthodoxie marxiste qui traite l'exploitation des femmes comme un vestige du féodalisme destiné à être liquidé une fois qu'elles auront été incorporées à la production capitaliste, Dalla Costa et James soulignent que l'exploitation des femmes garde une fonction centrale dans l'accumulation du capital tant que les femmes continuent d'être les agents de la production et de la reproduction de la force de travail. C'est déjà lorsqu'on commence à aborder le thème du travail domestique par rapport au mode de production capitaliste que s'impose une révision du concept de l'accumulation capitaliste, laquelle est définie par Marx comme le résultat de la rencontre entre les moyens de la production et la force de travail - une rencontre dont le résultat est la création de la plusvalue.

3 Cette révision, dont les lignes ont déjà été esquissées par Rosa Luxemburg ${ }^{3}$, nous permet d'aborder les conditions de l'accumulation générale qui sont totalement différentes de celles de l'accumulation individuelle. R. Luxemburg indique - en constatant le fait que 
Marx a élaboré le Capital sur la base de l'hypothèse d'une domination universelle et exclusive du mode de production capitaliste - que les conditions de l'accumulation générale ne peuvent être actualisées dans une société qui ne serait constituée que de capitalistes et d'ouvriers.

Par conséquent, il est erroné de considérer le mode de production capitaliste comme un rapport qui se déroule exclusivement entre ces deux groupes sociaux : il devrait bien plutôt être caractérisé à travers son rapport avec d'autres modes de production, étant donné que le processus à la fois souple et vertigineux de l'accumulation exige inévitablement la pénétration sans entraves dans ces autres modes appartenant à d'autres formes sociales, et surtout l'existence d'autres agents différents des capitalistes et des ouvriers afin de garantir la consommation.

D'après la démarche de Rosa Luxemburg, il est évident qu'il n'existe pas de société capitaliste "pure", entièrement dominée par le mode de production capitaliste: l'accumulation du capital a toujours besoin de s'approprier les autres modes de production, puisque la plus-value ne saurait être accumulée seulement par la vente aux capitalistes et aux ouvriers - il faut que la marchandise circule à travers des réseaux sociaux dont le mode de production n'est pas capitaliste. La puissance de l'accumulation du capital est donc étroitement liée à son expansion universelle vers l'appropriation des autres modes de production.

6 En ce sens, l'accumulation du capital est d'ores et déjà la destruction de ces autres modes de production, ce qui nous renvoie au concept de l'accumulation dite "primitive» esquissé par Marx dans le Livre I du Capital (die sogenannte ursprüngliche Akkumulation). D'abord, il faut rappeler que le travail domestique - qui se trouve toujours aux marges du mode de production capitaliste, mais qui rend possible en même temps la re/production de la force travail au sein de celui-ci - est l'un des moteurs de l'accumulation du capital. C'est ainsi qu'on pourra le traiter comme un des modes de production extérieurs au capitalisme, qui constituent en même temps les conditions de possibilité de l'accumulation du capital. Ce rôle joué par la division sexuelle du travail et par la naturalisation du travail domestique dans l'accumulation du capital nous permet de penser celle-ci comme un dispositif de colonisation intérieure.

7 Des auteurs comme Silvia Federici et Mariarosa Dalla Costa, parmi d'autres, consacrent leurs efforts à la mise-en-récit d'une opération matérialiste visant la démystification de tous les discours moralisants et naturalisants qui traitent le travail domestique comme une tâche maternelle à l'évidence incontestable; ainsi, elles s'interrogent sur le rôle du travail domestique dans le mode de production capitaliste. Le travail domestique, renvoyé à l'extérieur du mode de production capitaliste dans le jeu de l'identité abstraite du capital se définissant soi-même par l'appropriation de son autre, continue d'assurer les moyens de la reproduction du capital.

8 C'est justement dans ce sens qu'il faut repérer le rapport entre les différents modes de production, aussi bien que le lien entre les modes d'exploitation féodal, autrement dit patriarcal dans le cadre qui nous occupe, et capitaliste. Ces analyses autour du thème du travail domestique nous renvoient à une étude de l'accumulation dite primitive en tant qu'opérateur de la conceptualisation marxienne du processus de production des salariés et des capitalistes au début de l'époque moderne.

Suivant Louis Althusser et ses collaborateurs, il est possible et fécond de traiter le discours autour de l'accumulation dite primitive ou initiale comme une théorie de la 
transition d'un mode de production à un autre, voire même comme la théorie du processus de constitution d'un mode de production déterminé4.

10 C'est dans le même sens que Silvia Federici entame une discussion sur le changement radical de la position des femmes dans la société à cette époque, sur la mise en place d'un dispositif de contrôle strict et rigoureux sur le corps des femmes et leur pouvoir reproductif. La chasse aux sorcières, qui coïncide chronologiquement avec la colonisation, les enclosures en Angleterre, le début de l'esclavage dans les colonies, la mise en œuvre de lois pénales draconiennes, peut être traitée comme l'un des «effets » pris dans la causalité immanente ou métonymique de cette période dite de l'accumulation primitive. C'est cette possibilité d'aborder le lien entre le mode de production capitaliste et son extériorité dans le cadre du travail domestique qui permet d'interroger l'impact de la prolétarisation sur les femmes et la destruction de tout mode de subsistance d'une population massive, qui caractérisent cette période de l'accumulation dite primitive.

\section{L'accumulation dite primitive}

11 C'est dans le XXIVème chapitre du Tome I du Capital, que Marx commence à cerner le processus entraînant la production d'une population d'hommes qui n'ont rien d'autre que leur travail pour se maintenir en vie. Suivant Rosa Luxemburg, on peut dire que le chapitre porte en réalité sur l'appropriation des autres modes de production, bien que ces processus ne soient considérés par Marx que comme la genèse du capitalisme et que Marx réaffirme la domination universelle et exclusive de la production capitaliste dès qu'il commence à analyser la production capitaliste et sa circulation ${ }^{5}$.

Comme on l'a déjà fait remarquer, suivant l'interprétation de Rosa Luxemburg, nous constatons que l'accumulation du capital appartient à une toute autre nature que l'accumulation de la plus-value (Mehrwert) et qu'elle compte sur d'autres formes, d'autres strates et d'autres organisations sociales pour se soutenir.

La singularité de ce chapitre marxien a également attiré l'attention d'autres commentateurs. Jason Read constate ainsi que ce chapitre ne peut être traité seulement comme une critique de l'économie politique classique ou de l'histoire du capitalisme en Angleterre. En se référant à Antonio Negri et à Louis Althusser, il indique que le chapitre en question est surtout lié à la matérialité des relations sociales et à la subjectivité6. La théorie marxienne de l'accumulation dite primitive est donc un récit non-économiste de l'essor d'un mode de production; selon ce récit celui-ci ne désigne pas simplement une relation économique particulière qui a des effets linéaires sur les autres relations ${ }^{7}$. C'est en ce sens que Negri souligne, tout comme Althusser, le fait que les dimensions sociales de l'existence, qui sont généralement traitées comme des suppléments nécessaires du mode de production - l'Etat, les lois, les rapports de pouvoir, la constitution de la subjectivité - sont en réalité ses éléments constitutifs ${ }^{8}$. Une telle approche du mode de production nous permet de penser celui-ci aussi par rapport au mode de subjectivation.

Dans le XXIVème chapitre du Tome I du Capital, Marx commence son analyse de l'accumulation dite primitive en se confrontant à un problème qui semble être un cercle vicieux : la production capitaliste se présuppose elle-même continuellement. Le mode de production capitaliste est fondé sur la richesse concentrée du côté des capitalistes et sur une population qui n'a rien d'autre que son travail pour se maintenir : 
L'argent et la marchandise ne sont pas d'emblée capital, pas plus que ne le sont les moyens de production et de subsistance. Il faut qu'ils soient transformés en capital. Mais cette transformation elle-même ne peut avoir lieu que dans des circonstances déterminées dont le point de convergence est le suivant: il faut que deux sortes très différentes de propriétaires de marchandises se présentent face à face et entrent en contact, d'un côté les propriétaires d'argent, de moyens de production et de subsistance, pour qui il s'agit de valoriser par l'achat de la force de travail d'autrui la somme de valeur qu'ils possèdent; de l'autre côté, des travailleurs libres, vendeurs de leur propre force de travail et donc vendeurs de travail. Travailleurs libres en ce double sens qu'ils n'appartiennent pas plus eux-mêmes immédiatement aux moyens de production et de consommation, comme c'est le cas des esclaves, des serfs, etc. que les moyens de production ne leur appartiennent, comme c'est le cas pour le paysan indépendant, etc. qu'ils en sont au contraire détachés, libérés et affranchis ${ }^{9}$.

Ainsi, le problème général de ce chapitre s'organise autour des questions suivantes : d'où provient-elle cette richesse, et qu'est-ce qui constitue la différence originaire entre les propriétaires d'argent et les travailleurs libres, vendeurs de leur propre force de travail ? Cette division, conceptualisée par l'économie politique classique comme une division morale entre une « élite laborieuse » et une «bande de canailles fainéantes » est récusée par Marx qui l'associe au péché originel en théologie. L'économie politique classique, dans sa non-vision qui crée son propre objet à partir de son opération aveugle, dissimule même pour elle-même le fait que son objet, qui est l'objet de sa non-vision, est d'ores et déjà une production ${ }^{10}$. Cette production nous renvoie à la matérialité historique entrânant le déracinement d'une population massive privée de ses moyens de subsistance :

Dans l'histoire de l'accumulation initiale, les moments qui font époque sont tous les bouleversements qui servent de leviers à la classe capitaliste en formation; mais surtout ce sont les moments où de grandes masses d'hommes ont brusquement et violemment été arrachés à leurs moyens de subsistance et jetés, prolétaires hors-laloi, sur le marché du travail ${ }^{11}$.

Chacun sait que dans l'histoire réelle, le premier rôle est tenu par la conquête, l'asservissement, le crime et le pillage, en un mot, par la violence ${ }^{12}$.

Silvia Federici consacre le premier chapitre de Caliban and the Witch aux mouvements sociaux au Moyen Âge: les hérétiques, les Anabaptistes, les révoltes des paysans, et notamment les révoltes des paysans en Angleterre sous l'influence des doctrines de John Ball (1381) et les Guerres des Paysans en Allemagne qui se déroulent en 1476, ainsi que les révoltes des artisans dans les villes - par exemple, la révolte des tisserands à Bruges et à Ghent en 1378 qui se présente comme la première "dictature des prolétaires » dans l'histoire. Elle indique que les mouvement sociaux qui se sont développés dans ces domaines différents caractérisent la vie quotidienne du Moyen Âge : ils ont entraîné «l'Âge d'Or des Prolétaires européens » au XVème siècle, en réaction à la valorisation du travail après le déclenchement de la «Peste Noire ».

17 On peut tout à fait associer ce récit moral des économistes classiques, fondé sur la division entre une élite laborieuse et les fainéants, à l'image-souvenir présente chez les idéologues du pouvoir lorsqu'ils s'efforcent de conjurer la crainte de ces révoltes sociales au Moyen Âge, lesquelles visaient un renversement total des rapports hiérarchiques dans la vie quotidienne de cette époque. Dans l'Âge d'Or des prolétaires européens, les prolétaires en révolte étaient généralement représentés par les classes dirigeantes et leurs idéologues comme des paresseux qui ne voulaient pas travailler. 

les pratiques contre-révolutionnaires des classes dirigeantes visant l'institutionnalisation $\mathrm{du}$ capitalisme face aux mouvements sociaux. En soulignant les agencements politiques des mouvements de révolte de l'époque médiévale, il est possible d'interpréter le processus d'institutionnalisation $\mathrm{du}$ capitalisme comme l'intensification et la cristallisation des forces réactives dirigées, bien que d'une manière aléatoire et hétérogène, contre les forces actives incarnées par les mouvements sociaux et présentes dans l'escalade de la crise du travail. nous renvoie inévitablement aux travaux de Michel Foucault. C'est dans ce sens que Jason Read, en s'interrogeant sur le rapport entre Foucault et l'œuvre marxienne, souligne le fait qu'il est possible de trouver chez Foucault une approche du marxisme qui dépasse la position critique exposée dans l'« Histoire de la Sexualité $»^{15}$. C'est dans la conférence intitulée «Les Mailles du Pouvoir », donnée au Brésil en 1981, que Foucault met l'accent sur la détermination réciproque entre le pouvoir disciplinaire et l'ascension du capitalisme.

On peut trouver facilement entre les lignes du livre II du Capital une analyse, ou du moins l'esquisse d'une analyse, qui serait l'histoire de la technologie du pouvoir, tel qu'il s'exerçait dans les ateliers et dans les usines ${ }^{16}$. 


\section{La prolétarisation des femmes}

23

C'est dans ce contexte qu'il faut situer la dégradation de la condition des femmes, leur séparation (Trennung) d'avec les moyens d'existences traditionnels, leur exclusion des travaux socialement acceptables et la création d'une division du travail fondée sur la différence entre les sexes - tous ces processus caractérisent la période de la "transition » vers le capitalisme.

Dans le deuxième chapitre de Caliban et la sorcière, Federici étudie la contre-révolution des classes dirigeantes face à la crise de l'accumulation qui a éclaté au XIVème et au XVème siècle : elle a recours au concept de l'accumulation dite primitive pour définir, d'après Marx, la restructuration sociale et économique gérée par les classes dirigeantes de l'Europe protomoderne contre la crise de l'accumulation, qui se manifeste comme une tendance à la désaccumulation.

Cependant, elle souligne le fait que la séparation des ouvriers de leurs moyens de subsistance, la colonisation et l'esclavage n'étaient pas les seules stratégies utilisées pour obtenir la prolétarisation d'une population massive. Ce processus exigeait de remplacer les corps humains par des machines de travail et de soumettre les femmes à la reproduction de la force du travail. L'accumulation du capital était, par-là même, l'accumulation de divisions et de discriminations au sein de la classe ouvrière.

Federici constate qu'une fois que l'économie de subsistance qui prévaut au Moyen Âge a disparu, l'intégralité de la production et de la reproduction caractérisant les sociétés dans lesquelles la production ne visait que l'utilité immédiate s'écroule; les rapports gouvernant la production et la reproduction deviennent les porteurs de différentes relations sociales caractéristiquement sexistes. Dans le nouveau régime monétaire qui est fondé sur la production pour le marché capitaliste, la reproduction de la force de travail a fini par impliquer des conditions de plus en plus dégradées. Le travail domestique, la valeur économique de la reproduction de la force de travail et sa fonction dans l'accumulation du capital ont été mystifiés et rendus invisibles comme "des tâches naturelles » des femmes.

Les femmes ont été aussi exclues de la plupart des travaux salariés; même lorsqu'elles pouvaient travailler, elles gagnaient beaucoup moins que le salaire moyen des hommes. Ces développements historiques ont culminé avec l'institutionnalisation de la figure sociale et professionnelle de la «femme au foyer » à temps plein. La séparation entre la production des marchandises et la reproduction de la force de travail deviennent alors les instruments de l'accumulation de travail non compensé, qui ont, à leur tour, rendu possibles l'usage capitaliste des marchés et le salariat. Cette séparation entre la production et la reproduction a alors créé une classe prolétaire féminine.

En constatant que la paupérisation, la criminalisation et la révolte sont les éléments structurels de l'accumulation du capital, Federici souligne que la pénurie associée à la disette et à la famine a coïncidé avec la révolution des prix au XVIème siècle et a marqué cette époque protomoderne par contraste avec l'affluence du XIVème siècle.

C'est bien à cette époque où les rues fourmillaient de masses paupérisées que la discipline sociale devint le problème le plus urgent pour la classe dirigeante. Ce qui est caractéristique de ce dispositif disciplinaire est, selon Federici, le rôle central du contrôle de la procréation et le discours visant à l'accroissement de la population qui constitue à 
ce moment-là le premier élément du discours biopolitique. Ainsi, Federici indique que c'est essentiellement la crise de la population au XVIème et XVIIème siècle qui a fait de la procréation et de l'accroissement de la population des enjeux majeurs pour l'État: les obsessions autour de l'accroissement de la population ont grandi énormément avec la montée des politiques mercantilistes.

Dans ce contexte, à partir de la deuxième partie du XVIème siècle, les gouvernements européens ont commencé à sanctionner plus sévèrement les différents moyens de contraception ainsi l'avortement. De nouvelles régulations ont été appliquées pour empêcher les femmes enceintes d'abréger leur grossesse. Les femmes ont été obligées de déclarer toutes leurs grossesses, selon une loi royale mise en œuvre en 1556 en France.

$\mathrm{Au}$ XVIème et XVIIème siècle, les condamnations à mort de femmes pour cause d'infanticide et de sorcellerie devinrent de plus en plus nombreuses; suite à la marginalisation des sages-femmes, le contrôle des femmes sur leur propre fécondité déclina. Bercer l'enfant devint la seule fonction reconnue aux femmes dans la procréation et la naissance, alors que le contrôle de l'accouchement revint aux médecins hommes. Une nouvelle règle médicale prescrivant la priorité de la vie de l'enfant par rapport à celle de la mère fut institutionnalisée, alors que la procédure traditionnellement appliquée par les sages-femmes reconnaissait un ordre de priorité opposé.

Ces modes de régulation ont été exercés pendant deux siècles pour obliger les femmes à accoucher: mettant l'accent sur le fait que les femmes utilisaient diverses formes de contraceptions et avaient un contrôle total sur leur fertilité au Moyen Âge, Federici compare le destin des femmes en Europe avec celui des femmes esclaves dans les plantations américaines. Marie Mies et ses collaborateurs qualifient la dégradation de la condition des femmes dans le processus de l'accumulation primitive comme «la colonisation mise en œuvre à l'intérieur de l'Europe $»^{17}$.

En rappelant que l'accouchement et les variations des populations ne sont jamais automatiques et "naturels", Federici s'oppose à Marx qui, dans le premier tome du Capital, rattache la détermination de la surpopulation à «l'accroissement naturel de la population ", en impliquant que le développement capitaliste coïncide spontanément avec l'augmentation de la population. Cette augmentation est traitée par Marx comme un «effet naturel » du développement économique. Or Federici s'interroge sur ce point: pourquoi considérons-nous l'accouchement comme un «fait de la nature » et non pas comme un événement social déterminé par des rapports de pouvoir? En réalité, l'accouchement et les variations des populations sont loin d'être naturels. Federici indique que l'État, dans toutes les phases du développement capitaliste, a été obligé d'imposer des régulations pour augmenter ou diminuer la force de travail: ce qui caractérise ce processus, selon elle, est la transformation de la maternité en travail forcé et la condamnation des femmes à la procréation en faveur de l'État. Ces pratiques s'articulent avec un discours qui définit les femmes comme inutiles et dépendantes : il suffit de parcourir les discursivités autour de la "querelle des femmes », les discussions autour de la nature des femmes, pour comprendre l'émergence d'un nouveau dispositif caractéristiquement sexiste.

Les humanistes, les protestants réformés, les contre-révolutionnaires catholiques, tous s'accordaient pour dresser des femmes un portrait peu flatteur. L'idée que les femmes ne devaient pas travailler et qu'elles ne devaient qu'aider leurs époux devenait de plus en plus légitime et inscrite dans les lois, les registres d'impôt et la règlementation des guildes. Les gouvernements urbains conseillaient aux guildes d'ignorer le travail des 
femmes à la maison : tous les travaux gérés par les femmes dans la maison ont commencé à être considérés comme relevant du «ménage ». En même temps, la prostitution a augmenté énormément : au XVIème siècle, le viol aggravé n'était plus officiellement un crime. Federici situe tous ces «effets » dans le champ de la création de la figure de la "femme au foyer» et dans la restructuration de la famille comme centre de la reproduction de la force de travail. La famille en tant qu'elle est à la fois un équivalent du marché et l'instrument de la dissémination de la discipline capitaliste et de ce nouvel ordre patriarcal, devient, dans la période de l'accumulation primitive, l'institution la plus importante pour l'appropriation et la mystification du travail féminin. Toutefois, ce n'est qu'au XIXème siècle que la "famille moderne » fondée sur le travail reproductif non rétribué des femmes au foyer à plein temps a été institutionnalisée, tout d'abord en Angleterre (époque victorienne).

\section{La Chasse aux Sorcières}

C'est dans ce contexte témoignant de la dégradation radicale de la condition des femmes que des pratiques de violence extrême frappant la population féminine ont fait leur apparition : c'est l'époque de la chasse aux sorcières.

Le crime de maleficium (la magie noire) était inclus dans les lois des nouveaux royaumes germaniques au VIIème et VIIIème siècle. A cette époque, ce n'étaient que les arts magiques qui visaient les hommes et les biens qui étaient punis dans le cadre du maleficium. Vers la moitié du XVIème siècle, à l'âge de la crise du féodalisme, la sorcellerie devint l'un des plus grands crimes contre l'État, l'Église et la Nature.

Même à cette époque, les élites et l'aristocratie étaient sceptiques au sujet de la sorcellerie. C'est à partir du milieu du XVIème siècle que le nombre de femmes condamnées comme sorcières augmenta et que les tribunaux séculaires assumèrent la responsabilité d'organiser directement les persécutions. De 1580 à 1630, alors que les rapports féodaux étaient progressivement remplacés par les institutions du capitalisme mercantiliste, la chasse aux sorcières s'intensifiait d'une manière décisive. Federici met l'accent sur le fait que la chasse aux sorcières était une action politique mise en œuvre la plupart du temps par des juristes et des théologiens spécialistes en démonologie et qu'elle était soutenue par des intellectuels de l'époque comme Thomas Hobbes et Jean Bodin.

En rejoignant les travaux de Michael Taussig - lequel, dans son livre de 1980 intitulé The Devil and the Commodity Fetishism in South America, indique que la croyance aux doctrines démoniques augmente dans les périodes de transition d'un mode de production à un autre $^{18}$-, Federici associe cette violence, qui était principalement dirigée contre les femmes âgées dépourvues de tout moyen de subsistance, les sages-femmes et les guérisseuses, à la prolétarisation des femmes dans les conditions souvent extrêmes qu'on vient d'évoquer. Il est possible de repérer une continuité entre les massacres et les persécutions dirigés contre les hérétiques et ceux qui visaient les sorcières : la chasse aux sorcières était surtout dirigée contre les femmes des classes laborieuses sous prétexte de crimes liés à la procréation (comme l'infanticide, l'avortement etc.), de promiscuité et de crimes contre la propriété privée des biens. Cela montre que l'essor de la chasse aux sorcières devrait être considéré comme relevant de la causalité métonymique du processus d'accumulation primitive. 

caractérisée par l'oppression de la sexualité : ce qui marque alors le rapport des femmes avec la sexualité est la censure, la répression et les interdits. En se référant à l'analyse de Michel Foucault dans son "Histoire de la sexualité», Federici constate que cette « explosion du discours sur la sexualité » s'est manifestée d'une manière particulièrement évidente dans la chambre de torture destinée aux sorcières : elle s'oppose alors à Foucault et rappelle que ce « discours " proliférant n'est pas un dispositif alternatif par rapport à la censure, aux interdits sexuels et à la crainte de la sexualité. Au contraire, il fonctionne en s'articulant à ces interdits et à cette hantise : le but de la chasse aux sorcières était le rejet de la sexualité non-procréative. Les procès-verbaux des procès contre les sorcières contiennent des longues séries d'actes sexuels non-procréatifs. Un autre élément important dans ce domaine discursif est le fait que les femmes suspectées d'être des sorcières étaient associées avec l'animalité : il était généralement admis que ces femmes pouvaient prendre des formes animales. La violence exterminatrice exercée contre les femmes était donc discursivement nourrie par l'animalisation des femmes, selon une continuité qui reliait l'image féminine aux figures de l'hérétique, de l'enfant, du prolétaire, de l'insurgé et du sauvage.

Comme témoignage de cette continuité entre la figure de l'insurgée et de la sorcière, Federici cite les analogies et les rapprochements entre la figure de la sorcière et les mythologies autour des pétroleuses pendant la Commune de Paris en 1871. Les pétroleuses étaient décrites comme des vieilles femmes avec des yeux sauvages et des cheveux ébouriffés. Dans ces mythes qui étaient assez répandus dans la presse bourgeoise de l'époque, les femmes prolétariennes étaient suspectées de se promener dans les rues de Paris avec des récipients remplis de gazoline, prêtes à mettre le feu à toute la ville. De milliers de femmes caractérisées suivant un imaginaire très proche de celui de la chasse aux sorcières ont été exécutées comme pétroleuses.

En outre, il ne faut pas oublier que la chasse aux sorcières fut «exportée » vers les colonies. Irene Silverblatt souligne, dans Moon, Sun and Witches (1987) ${ }^{19}$, le lien entre la chasse aux sorcières au Pérou et la restructuration des rapports sexuels dans cette société colonisée. Dans le dernier chapitre de son livre, qui est consacré plus spécifiquement à la colonisation, Federici explique que les figures du sauvage, du cannibale et du barbare servirent aux ethnologues européens comme "modèles ethnographiques » dans leurs interprétations des cultures, des religions et des rapports sexuels au sein des populations colonisées.

Pour conclure : c'est dans cette perspective que le processus d'institutionnalisation de la famille bourgeoise peut être considéré comme le «corrélat » des enclosures des terres des enclosures menées dans le champ de la subjectivité - et que la dégradation, l'infantilisation, l'animalisation des femmes peuvent être comprises comme des opérations de "colonisation intérieure ». Il semble aujourd'hui que les pratiques de violence extrême qui furent la condition de possibilité de l'accumulation dite primitive en tant que "forces" hétérogènes qui ont créé le champ du mode de production capitaliste - continuent d'être les conditions structurelles de l'accumulation du capital. 


\section{NOTES}

1. Silvia Federici, Caliban et la sorcière. Femmes, corps et accumulation primitive, traduction de l'anglais (États-Unis) par le collectif Senonevero, revue et complétée par Julien Guazzini, Paris, Entremonde, 2014.

2. Mariarosa Dalla Costa-Selma James, The Power of Women \& the Subversion of the Community, Bristol, Falling Wall Press, 1972; Selma James, Sex, Race and Class, Bristol, Falling Wall Press, 1975.

3. Rosa Luxemburg, The Accumulation of Capital (1913), chap. XXVI, p.325, http:// www.marxists.org/archive/luxemburg/1913/accumulation-capital/index.htm.

4. Louis Althusser et al. Lire « Le Capital », Paris, Maspero, 1965.

5. R. Luxemburg, The Accumulation of Capital, op. cit., p. 332.

6. Jason Read, «Primitive Accumulation: The Aleatory Beginning of Capitalism », in Rethinking Marxism, 14, March 2007, p. 150.

7. Ibid. p. 151

8. Ibid.

9. Karl Marx, Le Capital, Livre I, Paris, Editions Sociales, 1982, p. 804.

10. L. Althusser et al. Lire « Le Capital », op. cit.

11. K. Marx, Le Capital, op. cit., p. 806.

12. İbid. p. 804.

13. J. Read, « Primitive Accumulation: The Aleatory Beginning of Capitalism », art. cit., p. 573

14. Ibid.

15. Ibid. p. 39

16. Michel Foucault, « Les Mailles du Pouvoir », in Dits et Ecrits, t. IV, 1980-1988, Paris, Gallimard, 2001, p. 1008.

17. Maria Mies, Patriarchy and Accumulation on a World Scale: Women in the International Divison of Labour, London \& New York, Zed Books, 1984; voir aussi Maria Mies, Veronica Bennholdt, Claudia Von Verlhof, Women: The Last Colony, London \& New York, Zed Books, 1988.

18. Michael Taussig, The Devil and Commodity Fetishism in South America, State of Carolina, University of Carolina Press, 1980.

19. Irene Silverblatt, Moon, Sun and Witches. Gender Ideologies and Class in Inca and Colonial Peru, Princeton, Princeton University Press, 1987.

\section{RÉSUMÉS}

L'article étudie les positions féministes-matérialistes exposées dans l'ouvrage de Silvia Federici et sa vision de l'accumulation originaire. 
INDEX

Thèmes : marxisme, féminisme, anthropologie

Index géographique : Europe

Mots-clés : accumulation, corps, sorcière, colonisation interne

Index chronologique : Epoque moderne

\section{AUTEUR}

\section{ÖZNUR KARAKASS}

Öznur Karakass étudie la philosophie à l'Université Oberta de Catalogne et à l'Université de Toulouse II « Jean Jaurès ». 